\title{
Evaluation of viral load thresholds for predicting new WHO Stage 3 and 4 events in HIV-infected children receiving highly active antiretroviral therapy
}

\author{
George K Siberry, MD, MPH${ }^{1}$, D. Robert Harris, $\mathrm{PhD}^{2}$, Ricardo Hugo Oliveira, $\mathrm{MD}^{3}$, Margot \\ R. Krauss, MD, MPH${ }^{2}$, Cristina B. Hofer, MD, $\mathrm{PhD}^{3}$, Adriana Aparecida Tiraboschi, $\mathrm{MD}^{4}$, \\ Heloisa Marques, MD $^{5}$, Regina C. Succi, MD, $\mathrm{PhD}^{6}$, Thalita Abreu, $\mathrm{MD}^{3}$, Marinella Della \\ Negra, MD, PhD ${ }^{7}$, Lynne M. Mofenson, MD ${ }^{1}$, and Rohan Hazra, MD ${ }^{1}$ for the NISDI PLACES \\ Protocol ${ }^{\star}$ \\ ${ }^{1}$ Pediatric Adolescent Maternal AIDS Branch, Eunice Kennedy Shriver National Institute of Child \\ Health and Human Development, National Institutes of Health, Bethesda, MD, USA \\ ${ }^{2}$ Westat, Rockville, MD, USA \\ ${ }^{3}$ Instituto de Puericultura e Pediatria Martagão Gesteira, Universidade Federal do Rio de Janeiro, \\ Rio de Janeiro, Brasil \\ ${ }^{4}$ Faculty of Medicine of Ribeirao Preto, University of São Paulo, São Paulo, Brasil \\ ${ }^{5}$ Faculty of Medicine of São Paulo, University of São Paulo, Brasil \\ ${ }^{6}$ Universidade Federal de São Paulo, UNIFESP, São Paulo, Brasil \\ ${ }^{7}$ Instituto de Infectologia Emilio Ribas, São Paulo, Brasil
}

\section{Abstract}

Background-This study evaluated a wide range of viral load (VL) thresholds to identify a cutpoint that best predicts new clinical events in children on stable highly-active antiretroviral therapy (HAART).

\begin{abstract}
Methods-Cox proportional hazards modeling was used to assess the adjusted risk of World Health Organization stage 3 or 4 clinical events (WHO events) as a function of time-varying CD4, VL, and hemoglobin values in a cohort study of Latin American children on HAART $\geq 6$ months. Models were fit using different VL cut-points between 400 and 50,000 copies $/ \mathrm{mL}$, with model fit evaluated on the basis of the minimum Akaike Information Criterion (AIC) value, a standard model fit statistic.
\end{abstract}

Results-Models were based on 67 subjects with WHO events out of 550 subjects on study. The VL cutpoints of $>2600$ copies $/ \mathrm{mL}$ and $>32,000$ copies $/ \mathrm{mL}$ corresponded to the lowest AIC values and were associated with the highest hazard ratios $[2.0(\mathrm{p}=0.015)$ and $2.1(\mathrm{p}=0.0058)$, respectively] for WHO events.

\footnotetext{
Corresponding Author: George K Siberry, MD, MPH, Medical Officer, Pediatric, Adolescent, and Maternal AIDS (PAMA) Branch, Eunice Kennedy Shriver National Institute of Child Health and Human Development, National Institutes of Health, 6100 Executive Boulevard, Room 4B11H, Bethesda, MD 20892-7510, (P) 301.496.7350; (F): 301.496.8678, siberryg @ mail.nih.gov.

Contact Information for Alternate Corresponding Author: D. Robert Harris, Senior Study Director/Senior Epidemiologist,

Westat, 1650 Research Boulevard, Rockville, MD 20850-3195, (P) 301.251.4267; (F) 301.279.4545, harrisr1 @ westat.com

Conflicts of Interest: All authors confirm that they have NO financial arrangement with any company whose product or competing product plays a role in the manuscript.
} 
Conclusions-In HIV-infected Latin American children on stable HAART, two distinct VL thresholds (> 2,600 copies $/ \mathrm{mL}$ and $>32,000$ copies $/ \mathrm{mL}$ ) were identified for predicting children at significantly increased risk of HIV-related clinical illness, after accounting for CD4 level, hemoglobin level, and other significant factors.

\section{Keywords}

Pediatric HIV infection; viral load monitoring; viral load threshold; Latin America

\section{Introduction}

The importance of quantitative HIV RNA, or viral load (VL), assays for monitoring the treatment of HIV infection is well established for adults and children and is considered standard practice in higher-resource settings [1,2]. VL prior to initiation of highly active antiretroviral therapy (HAART) has been established as an independent predictor of disease progression in children [3-5]. We previously demonstrated the value of VL as an independent predictor of new HIV-related WHO stage 3 and 4 events (WHO events) in a cohort of Latin American children on HAART [6]. In that study, most recent VL above the 5,000 copies $/ \mathrm{mL}$ level used in the WHO definition for virologic failure [7] was predictive of WHO events, independent of CD4-defined immunosuppression, hemoglobin level and other factors; in contrast, the commonly used VL threshold of $\geq 400$ copies $/ \mathrm{mL}$ was not an independent predictor of WHO events in that study The present analysis used the same data set to explore VL thresholds between 400 and 50,000 copies/mL to identify the cut-point for the best fitting model for predicting new WHO events in children on stable HAART ( 2 six months of continuous HAART).

\section{Methods}

Details of the NISDI (Eunice Kennedy Shriver National Institute of Child Health and Human Development [NICHD] International Site Development Initiative) pediatric protocol, eligibility criteria for this analysis and methods used when applying Cox proportional hazards modeling to assess the time to WHO events as a function of timevarying CD4-defined immunological status, VL, and hemoglobin level and time-fixed covariates have been published [6].

\section{Statistical Analysis}

The primary outcome measure was the first occurrence of a new WHO stage 3 or 4 event following the time of eligibility for this analysis. The analyses were based on fitting a proportional hazards regression model to the data repeatedly, each time using a different cutpoint for the HIV-1 RNA (viral load), with viral load (VL), CD4 immunosuppression and hemoglobin level treated as time-varying predictors. Additional covariates found to be independently associated with the occurrence of WHO events in the prior publication were also included in the modeling. The cut-point for the initial series of models was set at 500 copy intervals for VL up to 5,000 copies/mL and at 5,000 copy intervals for VL from 5,000 $-50,000$ copies $/ \mathrm{mL}$. A second series of models was fit to the data with cut-points varying by only 100 copies, from 2,000-3,000 copies $/ \mathrm{mL}$, and by 1,000 copies $/ \mathrm{mL}$ from 30,000 35,000 copies $/ \mathrm{mL}$, when honing in on the best fitting model for predicting WHO events. Model selection was based on the Akaike Information Criterion (AIC), for which the model having the minimum AIC value among competing models represents the best fit to the data [8]. All analyses were conducted using the SAS statistical software, version 9.0 (SAS Institute Inc. Cary, NC). 


\section{Results}

The models were based on 550 subjects, 67 who experienced a WHO stage 3 or 4 event during follow-up and 483 who were censored (did not experience an event during followup); there were an additional 22 subjects with WHO events who were excluded from the modeling due to missing values for one or more covariates, with most missing CD4 measures.

For each VL cut-point, the Table presents: 1) the AIC goodness of fit measure; 2) the estimated hazard ratio, confidence interval and $\mathrm{p}$-value for assessing the contribution of $\mathrm{VL}$ to the model; and 3) the p-values for assessing the contribution of CD4 immunosuppression and hemoglobin to the fitted model. The AIC started at a high point at the VL cut-point of 400 copies $/ \mathrm{ml}$, declined to the first low point occurring at a VL cut-point of $2600 \mathrm{copies} / \mathrm{ml}$ prior to rising again, and then declined to the second low point at a VL cut-point of 32,000 copies/mL (Table 1; Figure).

The hazard ratio (HR) provides an estimate of the instantaneous risk of experiencing a WHO event associated with having a VL at or above the specified cut-point. For example, the hazard ratio of 1.61 for the VL cut-point of 400 copies $/ \mathrm{mL}$ indicates that subjects who had a VL measure at or above this cut-point were $61 \%$ more likely to experience a WHO event as those whose VL did not reach this level. The risk (HR) of WHO events was higher for the VL cut-offs associated with the lowest AIC scores (ie, best fit to the data): subjects with VL at or above the cut-point of 2600 copies/mL had a 2.0-fold higher risk (HR) of WHO events compared to those whose VL was below this level ( $\mathrm{p}=0.015)$; subjects with VL at or above the cut-point of 32,000 copies/mL had a 2.1 higher risk (HR) of WHO events compared to those whose VL was below this level ( $\mathrm{p}=0.0058$ ). The HRs for CD4 level, HIV clinical stage, hemoglobin level, body size and age were very similar when using either VL cut-off of 2,600 or 32,000 copies $/ \mathrm{mL}$ in the model (Table 2).

\section{Discussion}

Prior analysis of this large cohort of perinatally HIV-infected Latin American children taking HAART for at least six months demonstrated that most recent VL greater than the WHO-defined virologic failure threshold of 5,000 copies $/ \mathrm{mL}$ - but not above the 6-month virologic failure threshold of 400 copies $/ \mathrm{mL}$ used by US pediatric treatment guidelines at the time - was an independent predictor of WHO stage 3 and 4 events, even after adjusting for most recent CD4-defined immunosuppression, hemoglobin level and other cofactors [6]. By repeatedly using the same proportional hazards regression modeling with different VL cutoffs, we were able to demonstrate that two distinct VL cut-offs ( 2,600 and 32,000 copies/ $\mathrm{mL}$ ) were the best fitting models for predicting WHO events among VL cut-offs between 400 and 50,000 copies/mL.

It was somewhat expected that this analysis would yield a VL cut-off close to the 5,000 copies $/ \mathrm{mL}$ threshold reported in the original analysis. It was surprising, however, that the search for the VL cut-off that added the most independent value for predicting HIV-related clinical events would also yield a second, much higher VL cut-off that had such similar performance characteristics in the model. Furthermore, there was no evidence that the similar performance of these two VL cut-offs in the model was due to compensatory differences between the HR for CD4 level, hemoglobin level, age and other factors included in the model The VL cut-off of 32,000 copies $/ \mathrm{mL}$ in the present analysis is similar to the 30,000 copies/mL threshold used in the PEN-PACT study that compared clinical and other outcomes among children randomized to switch to a new HAART regimen at a confirmed VL threshold of 1,000 copies/mL versus at a threshold of 30,000 copies/mL.[9] In that PEN- 
PACT study, the clinical outcomes for children who switched at 30,000 copies $/ \mathrm{mL}$ were similar to those for children who switched at the lower VL threshold. The finding in the current analysis of a maximum predictive value for clinical events when children had VL > $32,000 \mathrm{~mL}$ would be consistent with PEN-PACT findings showing no clinical detriment in waiting until $\mathrm{VL}$ of 30,000 copies $/ \mathrm{mL}$ to switch therapy in children.

Other factors besides HIV-related clinical events must also be considered when choosing a VL cut-off for treatment failure or as a trigger to switch to a new HAART regimen in children. In PEN-PACT, there was a trend in children on non-nucleoside reverse transcriptase inhibitor (NNRTI)-based therapy who switched therapy after a VL of 30,000 copies/mL to accumulate more resistance mutations to the nucleoside reverse transcriptase inhibitor (NRTIs) in the regimen. On the other hand, there may be fewer second-line antiretroviral drugs tested or in formulations appropriate for children, limiting the feasibility of making a switch to a new HAART regimen at low VL cut-offs. In resource-rich settings where virologic monitoring is routine, virologic failure is suspected when adults and children who have been on HAART for at least 6 months have repeatedly detectable VL measurements ( $\geq 50-200$ copies $/ \mathrm{mL}$ ) [1,2]. Assays that detect such low-level VL are unlikely to become available in low-resource settings. However quantitative RNA assays from dried blood spots have been developed for use in low-resource settings with detection limits that should allow for detecting children who have $\mathrm{VL}$ of 30,000 copies $/ \mathrm{mL}$ or even 2,600 copies/mL [10]. It will now be important to evaluate the clinical predictive value of a broad range of virologic cut-offs using data from other cohorts of children on stable HAART to help confirm the best target for virologic monitoring in pediatric HIV programs.

\section{Acknowledgments}

Sources of Funding: NIH

2011 NISDI PLACES Protocol Acknowledgement List: Principal investigators, co-principal investigators, study coordinators, data management center representatives, and NICHD staff include: Brazil: Belo Horizonte: Jorge

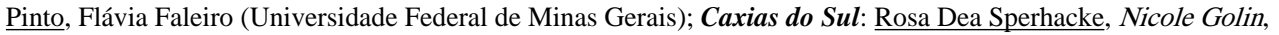
Sílvia Mariani Costamilan (Universidade de Caxias do Sul/ Serviço Municipal de Infectologia); Nova Iguacu: Jose Pilotto, Beatriz Grinsztejn, Valdilea Veloso, Luis Felipe Moreira, Ivete Gomes (Hospital Geral Nova de Iguacu HIV Family Care Clinic); Porto Alegre: Rosa Dea Sperhacke, Breno Riegel Santos, Rita de Cassia Alves Lira (Universidade de Caxias do Sul/Hospital Conceição); Rosa Dea Sperhacke, Mario Ferreira Peixoto, Elizabete Teles (Universidade de Caxias do Sul/Hospital Fêmina); Rosa Dea Sperhacke, Marcelo Goldani, Carmem Lúcia Oliveira da Silva, Margery Bohrer Zanetello (Universidade de Caxias do Sul /Hospital de Clínicas de Porto Alegre); Regis Kreitchmann, Marcelo Comerlato Scotta, Debora Fernandes Coelho (Irmandade da Santa Casa de Misericordia de Porto Alegre); Ribeirão Preto: Marisa M. Mussi-Pinhata, Maria Célia Cervi, Márcia L. Isaac, Fernanda Tomé Sturzbecher, Bento V. Moura Negrini (Hospital das Clínicas da Faculdade de Medicina de Ribeirão Preto da Universidade de São Paulo); Rio de Janeiro: Ricardo Hugo S. Oliveira, Maria C. Chermont Sapia (Instituto de Puericultura e Pediatria Martagão Gesteira); Esau Custodio Joao, Maria Leticia Cruz, Ana Paula Antunes, Jacqueline Anita de Menezes (Hospital dos Servidores do Estado); São Paulo: Regina Celia de Menezes Succi, Daisy Maria Machado (Escola Paulista de Medicina-Universidade Federal de São Paulo); Marinella Della Negra, Wladimir Queiroz, Yu Ching Lian (Instituto de Infectologia Emilio Ribas); Mexico: Mexico City: Noris Pavía-Ruz, Dulce Morales-Pérez, Jorge Gamboa-Cardeña (Hospital Infantil de México Federico Gómez); Peru: Lima: Jorge Alarcón Villaverde (Instituto de Medicina Tropical "Daniel Alcides Carrión”-Sección de Epidemiologia, UNMSM), María Castillo Díaz (Instituto Nacional de Salud del Niño), Mary Felissa Reyes Vega (Instituto de Medicina Tropical "Daniel Alcides Carrión" -Sección de Epidemiologia, UNMSM); Data Management and Statistical Center: Yolanda Bertucci, Laura Freimanis Hance, René Gonin, D. Robert Harris, Roslyn Hennessey, Margot Krauss, James Korelitz, Kathryn Miller, Sharon Sothern de Sanchez, Sonia K. Stoszek (Westat, Rockville, MD, USA); NICHD: Rohan Hazra, Lynne M. Mofenson, George K. Siberry (Eunice Kennedy Shriver National Institute of Child Health and Human Development, Bethesda, Maryland). Supported by NICHD Contract \# N01HD-3-3345 (2002-2007) and by NICHD Contract \# HHSN267200800001C (NICHD Control \#: N01HD-8-0001) (2007-2012). 


\section{References}

1. [Accessed October 21, 2011] Working Group on Antiretroviral Therapy and Medical Management of HIV-Infected Children; Guidelines for the Use of Antiretroviral Agents in Pediatric HIV Infection. Aug 11. 2011 p. 1-268. Available at http://aidsinfo.nih.gov/ContentFiles/PediatricGuidelines.pdf

2. Panel on Antiretroviral Guidelines for Adults and Adolescents. Guidelines for the use of antiretroviral agents in HIV-1-infected adults and adolescents. Department of Health and Human Services; Oct 14. 2011 p. 1-167.Available at http://www.aidsinfo.nih.gov/ContentFiles/AdultandAdolescentGL.pdf

3. Shearer WT, Quinn TC, LaRussa P, et al. Viral load and disease progression in infants infected with human immunodeficiency virus type 1. Women and Infants Transmission Study Group. N Engl J Med. 1997; 336(19):1337-42. [PubMed: 9134873]

4. Palumbo PE, Raskino C, Fiscus S, et al. Predictive value of quantitative plasma HIV RNA and CD4+ lymphocyte count in HIV-infected infants and children. JAMA. 1998; 279(10):756-61. [PubMed: 9508151]

5. Mofenson LM, Korelitz J, Meyer WA 3rd, et al. The relationship between serum human immunodeficiency virus type 1 (HIV-1) RNA level, CD4 lymphocyte percent, and longterm mortality risk in HIV-1-infected children. National Institute of Child Health and Human Development Intravenous Immunoglobulin Clinical Trial Study Group. J Infect Dis. 1997; 175(5): 1029-38. [PubMed: 9129063]

6. Oliveira R, Krauss M, Essama-Bibi S, Hofer C, Harris DR, Tiraboschi A, de Souza R, Marques H, Succi R, Abreu T, Della Negra M, Hazra R, Mofenson LM, Siberry GK. NISDI Pediatric Study Group 2010. Viral load predicts new world health organization stage 3 and 4 events in HIV-infected children receiving highly active antiretroviral therapy, independent of CD4 T lymphocyte value. Clin Infect Dis. 2010 Dec 1; 51(11):1325-33. [PubMed: 21039218]

7. Recommendations for a public health approach - 2010 Revision. World Health Organization; 2010. Antiretroviral therapy for HIV infection in children: Towards universal access. Available at: http://www.who.int/hiv/pub/paediatric/infants/en/index.html

8. Akaike H. Likelihood of a model and information criteria. Journal of Econometrics. 1981; 16:3-14.

9. Babiker A, Castronee Green H, Compagnucci A, Fiscus S, Giaquinto C, Gibb DM, Harper L, Harrison L, Hughes M, McKinney R, Melvin A, Mofenson L, Saidi Y, Smith ME, Tudor-Williams G, Walker AS. PENPACT-1 (PENTA 9/PACTG 390) Study Team. First-line antiretroviral therapy with a protease inhibitor versus non-nucleoside reverse transcriptase inhibitor and switch at higher versus low viral load in HIV-infected children: an open-label, randomised phase $2 / 3$ trial. Lancet Infect Dis. 2011; 11(4):273-83. [PubMed: 21288774]

10. Johannessen A, Garrido C, Zahonero N, et al. Dried blood spots perform well in viral load monitoring of patients who receive antiretroviral treatment in rural Tanzania. Clin Infect Dis. 2009; 49(6):976-81. [PubMed: 19663598] 


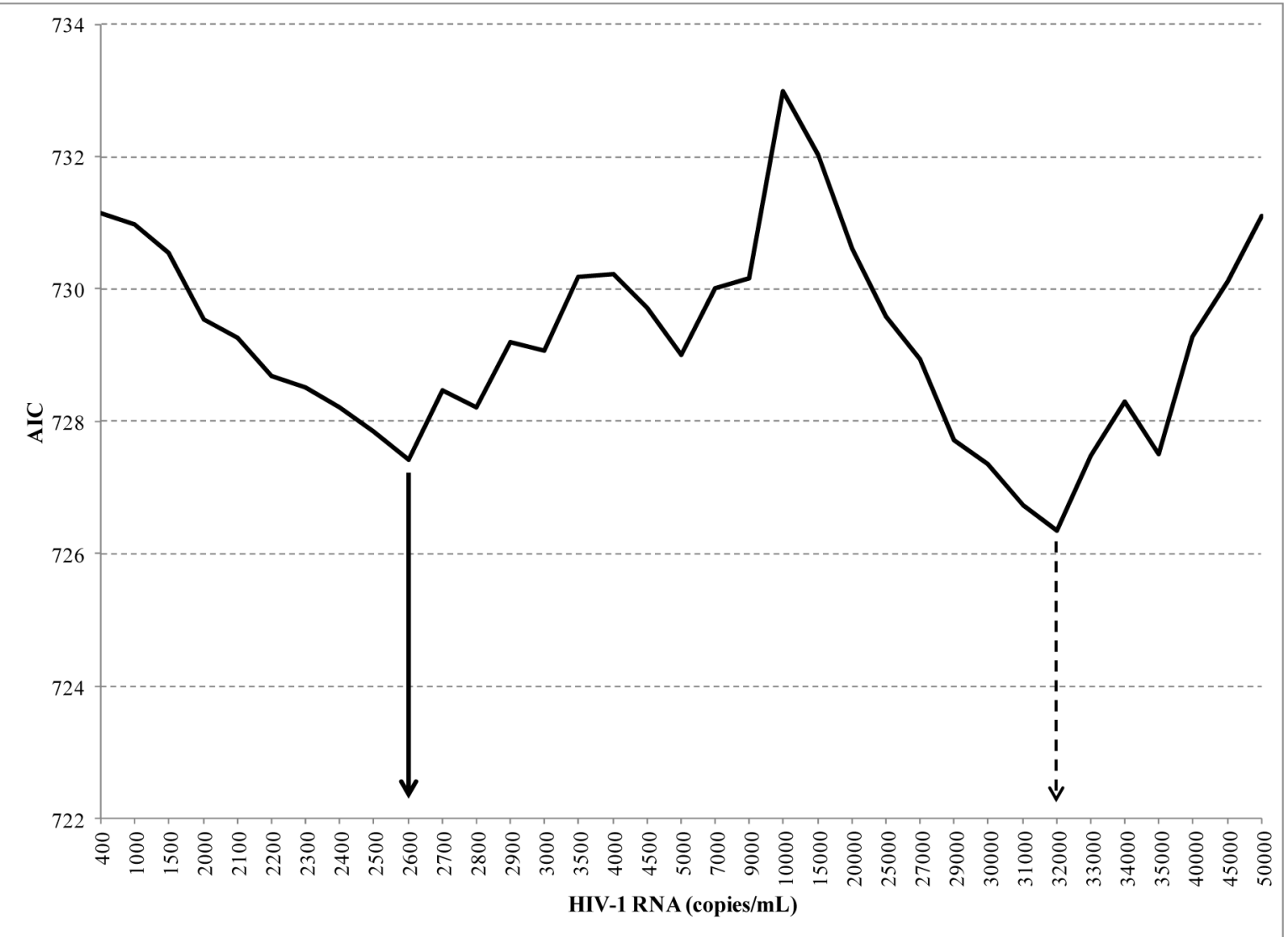

Figure 1.

Akaike Information Criterion (AIC) goodness-of-fit measure plotted as a function of HIV viral load cut-points 
(⿸丆口

0
0
0
0
0
0
0
0
0
0
0
0
0
0
00
0
0
0
0
0
0
0
0
0
0
0
0
0
0
0
0
0
0
0

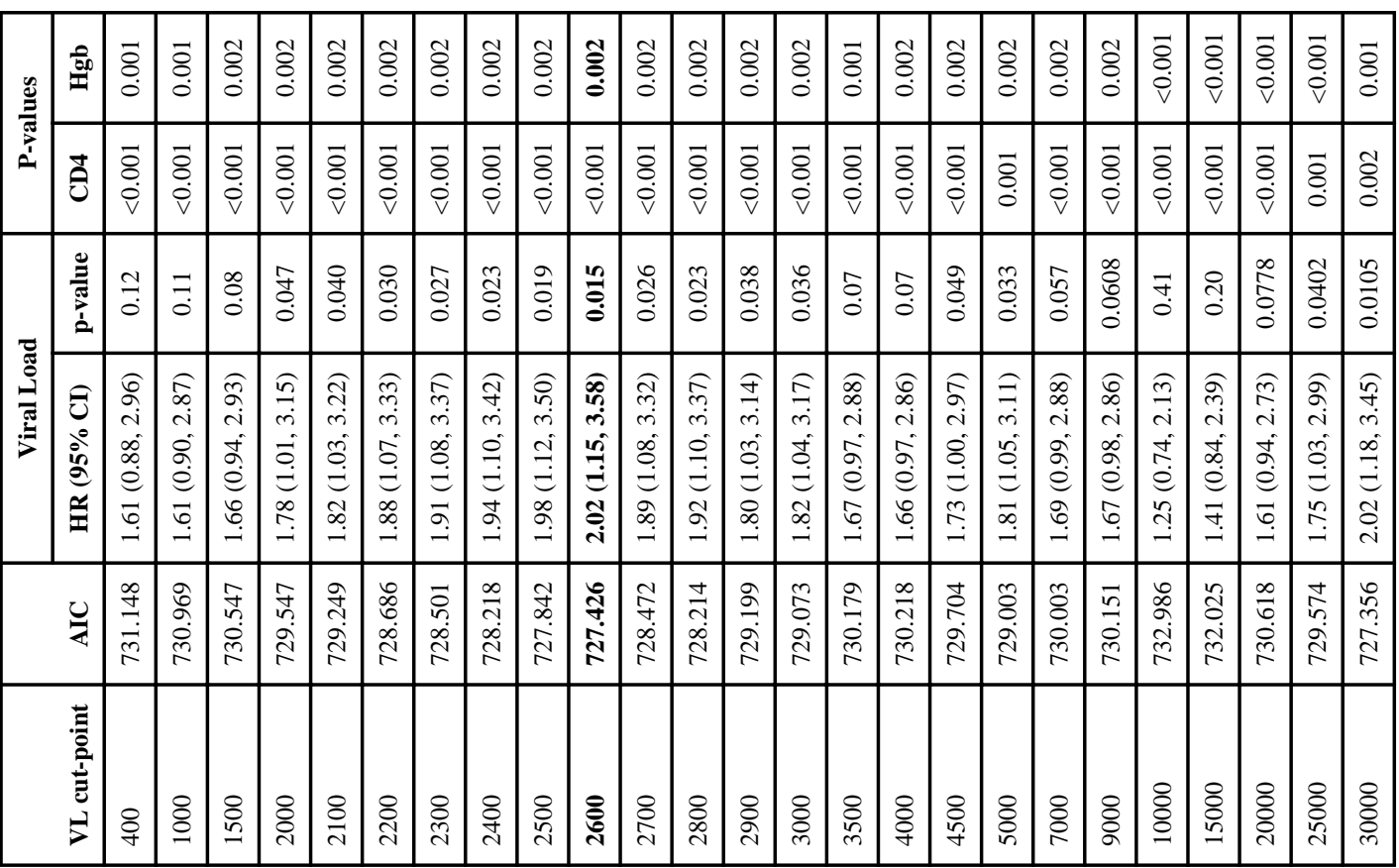




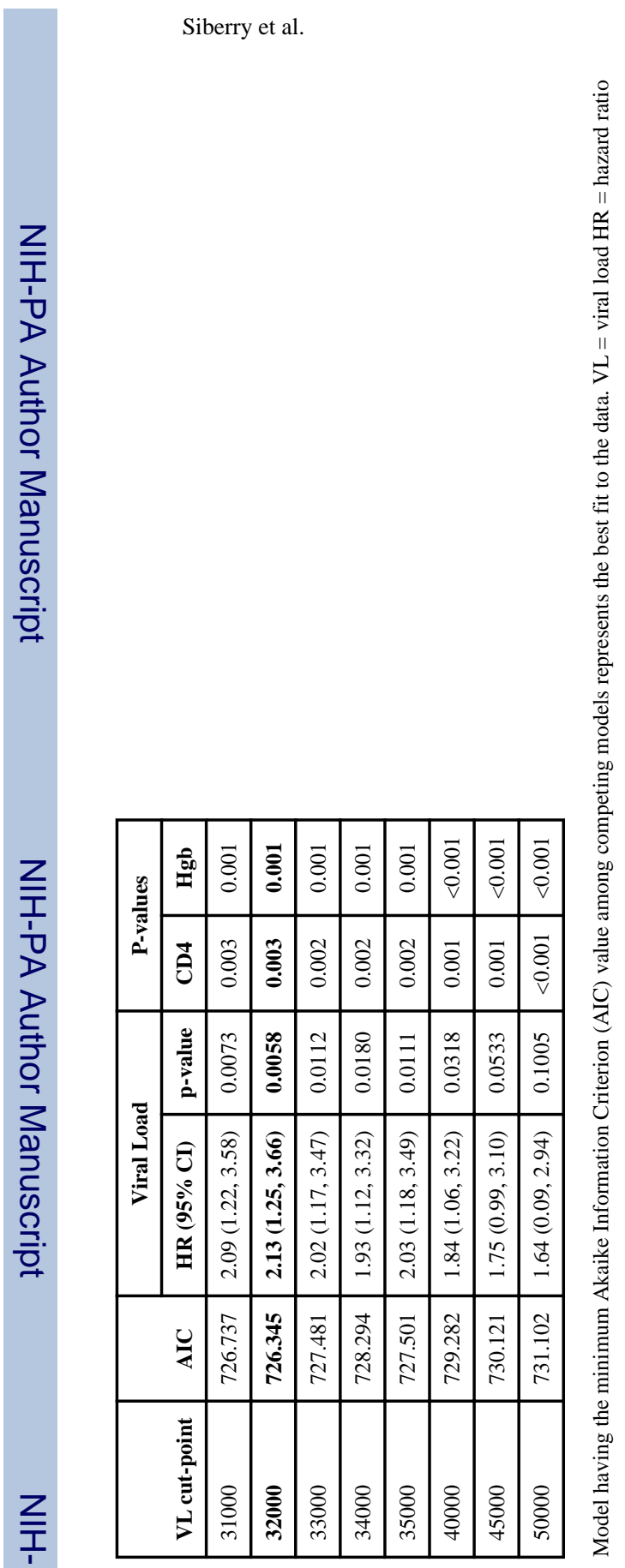

J Acquir Immune Defic Syndr: Author manuscript; available in PMC 2013 June 01. 
